\title{
Comparison of Free-Energy Methods to Calculate the Barriers for the Nucleophilic Substitution of Alkyl Halides by Hydroxide \\ DOI:
}

10.1021/acs.jpcb.0c02264

\section{Document Version}

Accepted author manuscript

Link to publication record in Manchester Research Explorer

Citation for published version (APA):

Ali, H. S., Higham, J., De Visser, S. P., \& Henchman, R. H. (2020). Comparison of Free-Energy Methods to Calculate the Barriers for the Nucleophilic Substitution of Alkyl Halides by Hydroxide. The Journal of Physical Chemistry B. https://doi.org/10.1021/acs.jpcb.0c02264

Published in:

The Journal of Physical Chemistry B

\section{Citing this paper}

Please note that where the full-text provided on Manchester Research Explorer is the Author Accepted Manuscript or Proof version this may differ from the final Published version. If citing, it is advised that you check and use the publisher's definitive version.

\section{General rights}

Copyright and moral rights for the publications made accessible in the Research Explorer are retained by the authors and/or other copyright owners and it is a condition of accessing publications that users recognise and abide by the legal requirements associated with these rights.

\section{Takedown policy}

If you believe that this document breaches copyright please refer to the University of Manchester's Takedown Procedures [http://man.ac.uk/04Y6Bo] or contact uml.scholarlycommunications@manchester.ac.uk providing relevant details, so we can investigate your claim.

\section{OPEN ACCESS}




\title{
Comparison of Free-Energy Methods to Calculate the Barriers for the Nucleophilic Substitution of Alkyl Halides by Hydroxide
}

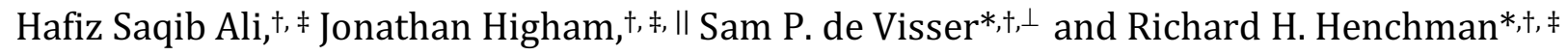 \\ † Manchester Institute of Biotechnology, The University of Manchester, 131 Princess Street, Manchester, M1 7DN, \\ United Kingdom \\ ‡ Department of Chemistry, The University of Manchester, Oxford Road, Manchester, M13 9PL, United Kingdom \\ ${ }^{\perp}$ Department of Chemical Engineering and Analytical Science, The University of Manchester, Oxford Road, Manchester, \\ M13 9PL, United Kingdom \\ II Institute of Genetics \& Molecular Medicine, The University of Edinburgh, Western General Hospital, Crewe Road South, \\ Edinburgh EH4 2XU, United Kingdom.
}

\begin{abstract}
Calculating the free energy barriers of liquid-phase chemical reactions with explicit solvent is a considerable challenge. Most methods use the energy and entropy (EE) of minimized single-point geometries of the reactants and transition state in implicit solvent using normal mode analysis (NMA). Explicit-solvent methods instead make use of the potential of mean force (PMF). Here we propose a new EE method to calculate the Gibbs free energy of reactants and transition states in explicit solvent by combining quantum mechanics/molecular mechanics (QM/MM) molecular dynamics simulations with Multiscale Cell Correlation (MCC). We apply it to six nucleophilic substitution reactions of the hydroxide transfer to methyl- and ethyl halides in water, where the halides are $\mathrm{F}, \mathrm{Cl}$ and $\mathrm{Br}$. We compare EE-MCC Gibbs free energy barriers using two Hamiltonians, self-consistent charge density functional based tight binding (SCC-DFTB) and B3LYP/6-31+G* Density Functional Theory (DFT) with respective PMF values, EE-NMA values using B3LYP/6-31+G* and M06/6-31G* DFT in implicit solvent and experimental values derived via Transition State Theory. The barriers using SCC-DFTB are found to agree well with PMF and experiment and previous computational studies, being slightly higher but improving on the lower values obtained for the implicit solvent. Achieving convergence over many degrees of freedom remains a challenge for EE-MCC in explicit-solvent QM/MM systems, particularly for the more expensive B3LYP/6-31+G* and M06/6-31G* DFT methods, but the insightful decomposition of entropy over all degrees of freedom should make EE-MCC a valuable tool for deepening the understanding of chemical reactions.
\end{abstract}

\section{INTRODUCTION}

Calculating the kinetics of chemical reactions has been a major ongoing target of theoretical and computational chemistry for many decades. ${ }^{1-3}$ This is a particular challenge in the liquid phase because it involves doing quantum mechanics calculations in a system comprising thousands of molecules. A number of different techniques have been developed for determining the kinetics of chemical reactions. The most common approach treats the reacting atoms by quantum mechanics, often at a high level, ${ }^{4,5}$ and approximates the solvent implicitly as a continuum with a dielectric constant and interfacial properties. ${ }^{6,7}$ Implicit-solvent models include the polarizable continuum model $(\mathrm{PCM}),{ }^{8}$ the self-consistant isodensity PCM (SCIPCM), ${ }^{9}$ the Onsager model ${ }^{10}$ or the universal solvent model (SMD). ${ }^{11}$ Similar to the treatment of gas-phase reactions, energies and Hessians are evaluated at the minima of the reactants and transition state, corresponding to absolute zero temperature, the internal vibrational energy and entropy at the temperature of interest are accounted for using normal mode analysis (NMA), the translational and rotational energy and entropy are calculated using the ideal-gas values, and rate constants are evaluated using Transition State Theory (TST). ${ }^{12,13}$ Energy may be understood via the many energy decomposition methods proposed ${ }^{14}$ while entropy is commonly interpreted in terms of normal modes for ideal gas molecules.

Two main advances have occurred beyond this standard implicit-solvent approach. First, explicit treatment of the surrounding non-reacting atoms was made possible by the Quantum Mechanics / Molecular Mechanics (QM/MM) method, ${ }^{15}$ which treats the small reacting region with $Q M$ and the rest of the system with MM. ${ }^{16}$ Second, moving away from single-point minimum calculations, extensive sampling of the thermalized ensemble was made possible with simulation 
methods such as molecular dynamics (MD) or Monte Carlo. While energy and enthalpy can be directly calculated from the average system Hamiltonian, existing entropy methods such as NMA are not easily applicable to multi-molecular systems, given the huge number of minima, ${ }^{17}$ meaning that the direct calculation of free energy from energy and entropy has not been possible for explicit-solvent systems. Other free-energy methods are used instead, such as Umbrella Sampling (US),$^{18}$ which yields the free energy as a function of the reaction coordinate, also known as the Potential of Mean Force (PMF). Quantitatively accurate free-energy barriers can be extracted from the PMF. Entropy barriers can be obtained using the restraint-release method, ${ }^{19}$ from the temperature dependence of the $\mathrm{PMF}^{20-23}$ or from the free-energy difference using a number of different formulations. ${ }^{24}$ However, these methods do not produce absolute entropy over all degrees of freedom and provide little direct understanding at the molecular level in the way that the energyentropy NMA method does in implicit-solvent. While energy typically dominates kinetics in gas-phase reactions, entropy may make a more comparable contribution in solvent or with catalysts, requiring it to be better understood.

In this work we seek to address this gap in capability of calculating and understanding the free energy barrier of a chemical reaction directly from the energy and entropy in explicit solvent. We compare implicit-solvent $\mathrm{QM}$ and explicit-solvent QM/MM methods to determine the reaction kinetics for the model reaction of second-order nucleophilic substitution of alkyl halides reacting with hydroxide $\mathrm{OH}^{-}$in water. Nucleophilic substitution, having equation $\mathrm{X}^{-}+\mathrm{RY} \rightarrow \mathrm{RX}+\mathrm{Y}^{-}$ where $X=$ halide, $R=$ alkyl group and $Y=$ leaving group, is an extensively studied reaction, ${ }^{25-30}$ via a single transition state with a concerted ligand switch. Most theoretical studies have been in the gas phase, ${ }^{25,26}$ others in implicit solvent, ${ }^{27,28}$ and more recent studies in explicit solvent. ${ }^{31,32}$ We employ four different Hamiltonians and three free-energy methods. The four Hamiltonians are QM density functional theory (DFT) with the B3LYP functional (HIB) and M06 (HIM) in implicit water solvent, $\mathrm{QM} / \mathrm{MM}$ with self-consistent charge density functional based tight-binding (SCC-DFTB) ${ }^{33,34}$ in explicit solvent (HES) and QM/MM with B3LYP in explicit solvent (HEB). The first free-energy method, which we designate the Energy Entropy Normal Mode Analysis (EE-NMA), uses the standard energy and entropy evaluated using NMA added to the energy minimum of the reactant encounter complex (REC) and transition state (TS). The second method uses the barrier of the PMF along a pre-defined reaction coordinate. The third method, newly proposed here and termed Energy Entropy Multiscale Cell Correlation (EE-MCC) calculates the barrier from the energy and entropy of the REC and TS, where the TS is taken as the maximum of the PMF. The energies are calculated from the average of the simulation Hamiltonian and the entropies are calculated using MCC. ${ }^{35,36}$ Experimental rate constants for the methyl halide reactions are converted into free energy barriers using TST as a point of comparison. Both PMF methods are found to give the best agreement with experiment, the SCC-DFTB being slightly better than B3LYP. EE-MCC SCC-DFTB values are in satisfactory agreement, performing better than EE-NMA B3LYP but EE-MCC B3LYP values could not be converged. EE-MCC is affected by statistical noise arising from having to calculate free energy of all molecules combined with the limited sampling possible in a multimolecular QM/MM system, but it provides substantial detail about the entropic contributions of every degree of freedom of the system.

\section{METHODS}

Systems of Interest. Six nucleophilic substitution reactions in water are considered. Hydroxide displaces the halide atom of $\mathrm{CH}_{2} \mathrm{XY}$, where $\mathrm{X}=\mathrm{F}, \mathrm{Cl}$ or $\mathrm{Br}$ and $\mathrm{Y}$ $=\mathrm{H}$ or $\mathrm{CH}_{3}$. Assuming that the binding of reactants is not rate-determining, we consider the reactant encounter complex (REC) which reacts in a unimolecular process via the transition state (TS) to the product alcohol and halide ion as shown in Scheme 1:

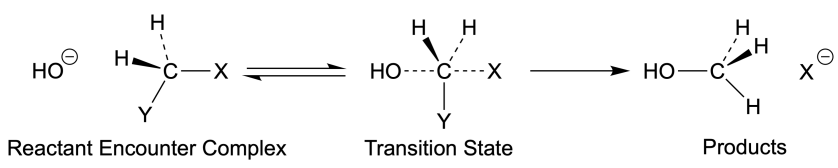

Scheme 1. Reaction mechanism of the nucleophilic substitution of the alkyl halide $\mathrm{CH}_{2} \mathrm{XY}$ where $\mathrm{X}=\mathrm{F}, \mathrm{Cl}$, $\mathrm{Br}$ and $\mathrm{Y}=\mathrm{H}, \mathrm{CH}_{3}$ with hydroxide.

\section{System Hamiltonians:}

HIB : QM DFT B3LYP in Implicit Solvent. The REC in implicit solvent is illustrated in Figure 1.

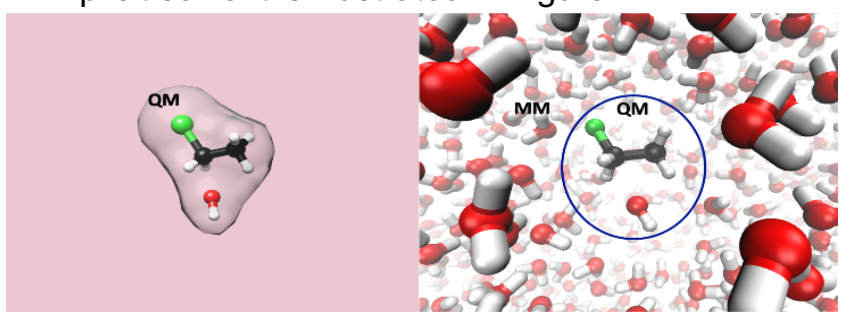

Figure 1. QM and MM regions for the implicit-water QM (left) and explicit-water QM/MM simulations (right). 
The B3LYP ${ }^{37}$ DFT method with the $6-31+G^{*}$ basis set was used. The Integral Equation Formalism variant of the Polarizable Continuum Model (IEFPCM) ${ }^{7}$ was used to model the solvent water. The solute molecules were built with GaussView for the REC. Minima and transition states were located by full geometry optimizations assisted by initial constraint geometry scan calculations in the Gaussian 09 software package. ${ }^{38}$

HIM: QM DFT M06 in Implicit Solvent. This is the same as HIB except that the M06 ${ }^{39,40}$ DFT method with the $6-31+G^{*}$ basis set was used.

\section{HES: QM/MM SCC-DFTB in Explicit Solvent.}

The alkyl halide and hydroxide comprise the QM region where the chemical reaction takes place and the water solvent comprises the MM region, as illustrated in Figure 1. The QM region was modeled with the Self-Consistent Charge Density Functional based Tight-Binding $(\mathrm{SCC}-\mathrm{DFTB})^{33}$ method implemented in AMBER $16 .{ }^{41}$ SCC-DFTB is less expensive than other methods such as B3LYP and has good accuracy for structure and relative energy. ${ }^{42}$ The AMBER formulation contained the necessary parameters for organic molecules ${ }^{43}$ and the halogen-related parameters ${ }^{44}$ were taken from the DFTB website. ${ }^{45}$ The MM region comprises explicit solvent TIP3P water molecules. ${ }^{9}$ The reactants interact with the MM region using the fixed van der Waals parameters from the Generalized AMBER Force Field $(G A F F)^{46}$ generated by Antechamber ${ }^{47}$ and variable configuration-specific RESP (Restrained Electrostatic Potential) charges for the electrostatic interaction using the AM1-BCC method. The reactant solute molecules were solvated with 1500 water molecules in a cubic 40 $\AA$ box using the xleap module of AMBER. ${ }^{41}$

Minimization of the REC of each system was done with 500 steps of steepest-descent minimization, followed by a 100 ps NVT (constant number, volume temperature) MD simulation and a 100 ps NPT simulation (constant pressure) with a 1 ps time constant. The resulting structure was used as the starting structure in each window in the US simulations, to be discussed in the Free Energy Methods section, enabling the simulations of each window to be run in parallel. For each window, there were 2000 steps of minimization, 100 ps of NPT MD equilibration, and $1 \mathrm{~ns}$ of MD data collection for the PMF calculations. Taking the TS at the maximum in this PMF, the REC and TS windows were each run for 10 ns to provide better sampling for the EE method. Forces and coordinates were saved every 1 ps. The simulations were performed at 1 bar pressure using the Berendsen barostat ${ }^{48}$ and $298.15 \mathrm{~K}$ temperature using the Langevin thermostat. Periodic boundary conditions were used together with a non-bonded cut-off of $8 \AA$, particle-mesh Ewald with default AMBER parameters, and a time step of $1 \mathrm{fs}$.

HEB: QM/MM B3LYP DFT in Explicit Solvent. The same QM method was used as in HIB, namely DFT with the B3LYP hybrid density functional and $6-31+G^{*}$ basis set for all atoms. This is implemented in AMBER with Gaussian as an external tool for the QM part. ${ }^{49}$ The same GAFF-TIP3P MM force field and simulation protocol are used as in HEB, except that 10 ps of sampling was used for data collection in the US simulations because this is a much slower and computationally demanding method.

\section{Free Energy Methods}

Energy Entropy-Normal Mode Analysis (EE-NMA). The Gibbs free energy barrier was calculated using

$$
\Delta G=H_{\mathrm{TS}}-H_{\mathrm{REC}}-T\left(S_{\mathrm{TS}}-S_{\mathrm{REC}}\right)
$$

where $T$ is the temperature and $H_{\mathrm{TS}}$ and $H_{\mathrm{REC}}$ are the enthalpies of the TS and REC, and $S_{\mathrm{TS}}$ and $S_{\mathrm{REC}}$ are the corresponding entropies. For an ideal-gas molecular complex at the level of molecular translation and rotation, all but the rotational entropy cancels in the difference between REC and TS, which is given by

$$
S_{\mathrm{rot}}=\frac{8 \pi^{2}}{\sigma} \prod_{i=x, y, z} \frac{h}{\sqrt{2 \pi I_{i} k_{\mathrm{B}} T}}
$$

where $\sigma$ is the symmetry number, taken as 1 for all reactions, $h$ is Planck's constant, $I_{i}$ are the moments of inertia for the three principal axes $x, y, z$, and $k_{\mathrm{B}}$ is Boltzmann's constant. The equations for the intramolecular enthalpy and entropy of the REC are

$$
H=E_{0}+P V+\sum_{i=1}^{3 N-6} h v_{i}\left(\frac{1}{2}+\frac{1}{\mathrm{e}^{\frac{h v_{i}}{k_{\mathrm{B}} T}}-1}\right)
$$

and

$$
S=\sum_{i=1}^{3 N-6}\left(\frac{h v_{i}}{T} \frac{1}{\mathrm{e}^{\frac{h v_{i}}{k_{\mathrm{B}} T}}-1}-k_{\mathrm{B}} \ln \left(1-\mathrm{e}^{-\frac{h v_{i}}{k_{\mathrm{B}} T}}\right)\right)
$$

where $E_{0}$ is the energy of the minimum, $P$ is pressure, $V$ is volume, $N$ is the number of atoms, and $v_{i}$ are the $3 N-6$ vibrational frequencies, which are calculated using NMA in Gaussian 09. The six lowest frequencies, 
being whole-molecule translations and rotations, are excluded, having already been accounted for. The same procedure is used for the TS except that there are $3 N-7$ frequencies because the imaginary frequency along the reaction coordinate is excluded. The ideal gas value $P V=k_{\mathrm{B}} T$ used by Gaussian cancels between the unimolecular REC and TS.

Potential of Mean Force (PMF). The PMF along the reaction coordinate $\xi$ is given by:

$$
\operatorname{PMF}(\xi)=-k_{\mathrm{B}} T \ln p(\xi)
$$

where $p(\xi)$ is the probability distribution of the system along $\xi$, which for all reactions is defined to be $\xi=$ $R_{\mathrm{C}-\mathrm{X}}-R_{\mathrm{C}-\mathrm{O}}$, where $R_{\mathrm{C}-\mathrm{X}}$ and $R_{\mathrm{C}-\mathrm{O}}$ are the $\mathrm{C}-\mathrm{X}$ and $\mathrm{C}-\mathrm{O}$ bond lengths respectively. $p(\xi)$ was evaluated using Umbrella Sampling (US). $\xi$ was divided into 31 windows separated by a $0.1 \AA$ spacing over the range of $-1.5 \AA$ to $1.5 \AA$. The system was restrained at each value of $\xi$ using a harmonic potential

$$
U_{i}(\xi)=k_{\mathrm{US}}\left(\xi-\xi_{i}^{0}\right)^{2}
$$

with a force constant $k_{\mathrm{US}}$ of $300 \mathrm{kcal} \mathrm{mol}{ }^{-1} \AA^{-2}$ for all reactions. ${ }^{50,51}$ The associated Gaussian distribution has a standard deviation of $0.03 \AA$, which is sufficient to span each window. MD simulations were used to generate probability distributions of $\xi$ for each window, which were converted to the full probability distribution $p(\xi)$ using the weighted histogram analysis method (WHAM). ${ }^{52,53} \Delta G$ was calculated as

$$
\Delta G=\operatorname{PMF}\left(\xi_{\mathrm{TS}}\right)-\operatorname{PMF}\left(\xi_{\mathrm{REC}}\right)
$$

Energy Entropy-Multiscale Cell Correlation (EEMCC). The Gibbs free energy barrier is evaluated from the enthalpy and entropy using Equation 1 as in EENMA plus the Gibbs free energy $\Delta G_{\mathrm{R}}$ for adding the restraint on the REC, which is given by

$$
\Delta G_{\mathrm{R}}=k_{\mathrm{B}} T \ln \left\langle\exp \left[U_{\mathrm{REC}}(\xi) / k_{\mathrm{B}} T\right]\right\rangle_{\xi}
$$

The TS is at $\xi$ of the maximum in the HES and HEB PMFs. The enthalpy is evaluated using

$$
\begin{gathered}
H=E_{\mathrm{QM}}+K_{\mathrm{MM}}+U_{\mathrm{MM}}+U_{\mathrm{QM} / \mathrm{MM}}+P V+ \\
\sum_{i=1}^{N_{\mathrm{vib}}} h v_{i}\left(\frac{1}{2}+\frac{1}{\mathrm{e}^{\frac{h v_{i}}{k_{\mathrm{B}} T}}-1}\right)-N_{\mathrm{vib}} k_{\mathrm{B}} T
\end{gathered}
$$

where $E_{\mathrm{QM}}$ is the average energy of the $\mathrm{QM}$ region, $K_{\mathrm{MM}}$ and $U_{\mathrm{MM}}$ are the average kinetic and potential energy of the $\mathrm{MM}$ region, and $U_{\mathrm{QM} / \mathrm{MM}}$ is the average interaction energy between the $\mathrm{QM}$ and $\mathrm{MM}$ regions. In the harmonic approximation, the classical energy $k_{\mathrm{B}} T$ is subtracted off for all $N_{\text {vib }}$ vibrational degrees of freedom and replaced with the quantum energy of a harmonic oscillator with frequency $v_{i}$, with the exception of the restrained reaction coordinate of the TS, for which the quantum energy is not included. Frequencies $v_{i}$ are calculated using $\mathrm{MCC}^{35,36}$ at two length scales: the molecule level and the united-atom level, where a united atom is each heavy atom together with any bonded hydrogens, treated as a rigid body. Note that this approach ignores the negligible entropy in high-frequency covalent bonds involving hydrogen atoms. Three vibrations are whole-molecule translations and three are whole-molecule rotations, termed "transvibrational" and "rovibrational". Their frequencies are derived from the eigenvalues $\lambda_{i}$ of the mass-weighted force covariance matrix and moment-of-inertiaweighted torque covariance matrix for the three principal axes of the solute complex using

$$
v_{i}=\frac{1}{2 \pi} \sqrt{\frac{\lambda_{i}}{k_{\mathrm{B}} T}}
$$

There are also $3 N-6$ internal vibrations that relate to translation of the $N$ united atoms. Their frequencies are evaluated using Equation 10 with the eigenvalues of the mass-weighted force covariance matrix. The remaining vibrations are rotations of the united atoms, being three if non-linear, two if linear, and none if a point. All matrices are constructed and diagonalized from the force and coordinate trajectories and connectivity information in the topology file using in-house $\mathrm{C}++$ code.

$S$ is calculated using Equation 4 with the same vibrational frequencies as for the vibrational energy at molecule and united-atom levels. For the TS the entropy of the vibration along the restrained reaction coordinate is excluded. The translational and rotational entropy of the solvent water is evaluated in the same way as the solute, except that the force and torque covariance matrices are averaged over all water molecules, and the total entropy is multiplied by the number of water molecules.

The rotational topographical entropy, relating to the number of solute orientations and termed "rotopographical", is calculated for the REC and TS with the equation ${ }^{35,36}$ 


$$
S=k_{\mathrm{B}} \sum_{N_{\mathrm{c}}} p\left(N_{\mathrm{c}}\right) \ln \left[\max \left(1, \frac{\left(N_{\mathrm{c}}^{3} \pi\right)^{1 / 2}}{\sigma}\right)\right]
$$

where, $p\left(N_{\mathrm{c}}\right)$ is the probability distribution of water coordination number $N_{\mathrm{c}}$ of the solute complex, and $\sigma$ is its symmetry number, taken as 1 for all reactions. The term inside the logarithm is the number of solute orientations, all assumed to have equal probability. $N_{\mathrm{c}}$ is evaluated with the Relative Angular Distance (RAD) $)^{54,55}$ algorithm using the centres of mass of the solute complex and each water molecule. A similar procedure is used for the rotational topographical entropy of the solvent, with $p\left(N_{\mathrm{c}}\right)$ being averaged over all water molecules, $\sigma=2$, and the number of orientations is divided by 4 to account for orientational correlations of hydrogen-bonded neighbours. ${ }^{36}$ Translational topographical entropy is omitted, being constant and canceling between the REC and TS.

Transition State Theory (TST). Experimental rate constants $k_{\text {expt }}$ from their Arrhenius parameters at $298 \mathrm{~K}$ for the nucleophilic substitution of methyl halides by hydroxide were converted into $\Delta G$ using the unimolecular TST equation ${ }^{26,56}$

$$
k_{\text {expt }}=\frac{k_{\mathrm{B}} T}{h} \exp \left(\frac{-\Delta G}{k_{\mathrm{B}} T}\right)
$$

This assumes that the chemical step of the reaction (Scheme 1 ) is rate-determining and that $k_{\text {expt }}$ is not influenced by the rate of reactant binding. ${ }^{56}$

\section{RESULTS}

Gibbs Free Energy Barriers. $\Delta G$ values are given in Table 1 for the HIB and HIM Hamiltonians using the EE-NMA method, both HES and HEB Hamiltonians using the PMF method, the HES Hamiltonian using the EE-MCC method, and experimental rate constants converted into $\Delta G$ using TST. See Supplementary Information for details of the error calculation for $\Delta G_{\mathrm{EE}-\mathrm{MCC}}^{\mathrm{HES}}$. The value of $\xi$ at the TS is not known from experiment but its $\Delta G$ value is placed at the HES PMF maximum for convenience. The EE-MCC $\Delta G$ values for the HEB simulations are not included because the associated energies and entropies were not converged over 10 ps. Figure 2 shows the HES and HEB PMFs for all reactions and both EE Gibbs free energies of the TS relative to the REC. The HES EE-MCC $\Delta G$ values take the TS at the maximum of the corresponding HES PMF, which for each reaction are at $\xi=-0.1,0.3,0.5$, $-0.1,0.2$ and $0.5 \AA$ respectively.

Table 1. Gibbs Free Energy Barriers for Each Reaction and Method versus Experiment (kcal mol-1)

\begin{tabular}{lcccccc}
\hline & \multicolumn{2}{c}{ Implicit Water } & \multicolumn{3}{c}{ Explicit Water } & Experiment/TST ${ }^{57,58}$ \\
& $\Delta G_{\mathrm{EE}-\mathrm{NMA}}^{\mathrm{HIB}}$ & $\Delta G_{\mathrm{EE}-\mathrm{NMA}}^{\mathrm{HIM}}$ & $\Delta G_{\mathrm{PMF}}^{\mathrm{HES}}$ & $\Delta G_{\mathrm{PMF}}^{\mathrm{HEB}}$ & $\Delta G_{\mathrm{EE}-\mathrm{MCC}}^{\mathrm{HES}}$ & $\Delta G$ \\
\cline { 2 - 6 } $\mathrm{CH}_{3} \mathrm{~F}$ & 16.4 & 15.1 & 20.0 & 18.4 & $21.6 \pm 1.2$ & $25.9 \pm 0.1$ \\
$\mathrm{CH}_{3} \mathrm{Cl}$ & 10.1 & 9.9 & 22.3 & 17.0 & $17.2 \pm 1.2$ & $24.5 \pm 0.1$ \\
$\mathrm{CH}_{3} \mathrm{Br}$ & 7.5 & 8.7 & 19.1 & 15.3 & $31.0 \pm 1.2$ & $22.7 \pm 0.2$ \\
$\mathrm{C}_{2} \mathrm{H}_{5} \mathrm{~F}$ & 20.5 & 20.2 & 21.0 & 17.6 & $25.9 \pm 1.2$ & - \\
$\mathrm{C}_{2} \mathrm{H}_{5} \mathrm{Cl}$ & 10.5 & 11.9 & 18.4 & 14.8 & $22.1 \pm 1.2$ & - \\
$\mathrm{C}_{2} \mathrm{H}_{5} \mathrm{Br}$ & 10.1 & 10.9 & 18.5 & 11.3 & $31.6 \pm 1.2$ & - \\
\hline
\end{tabular}



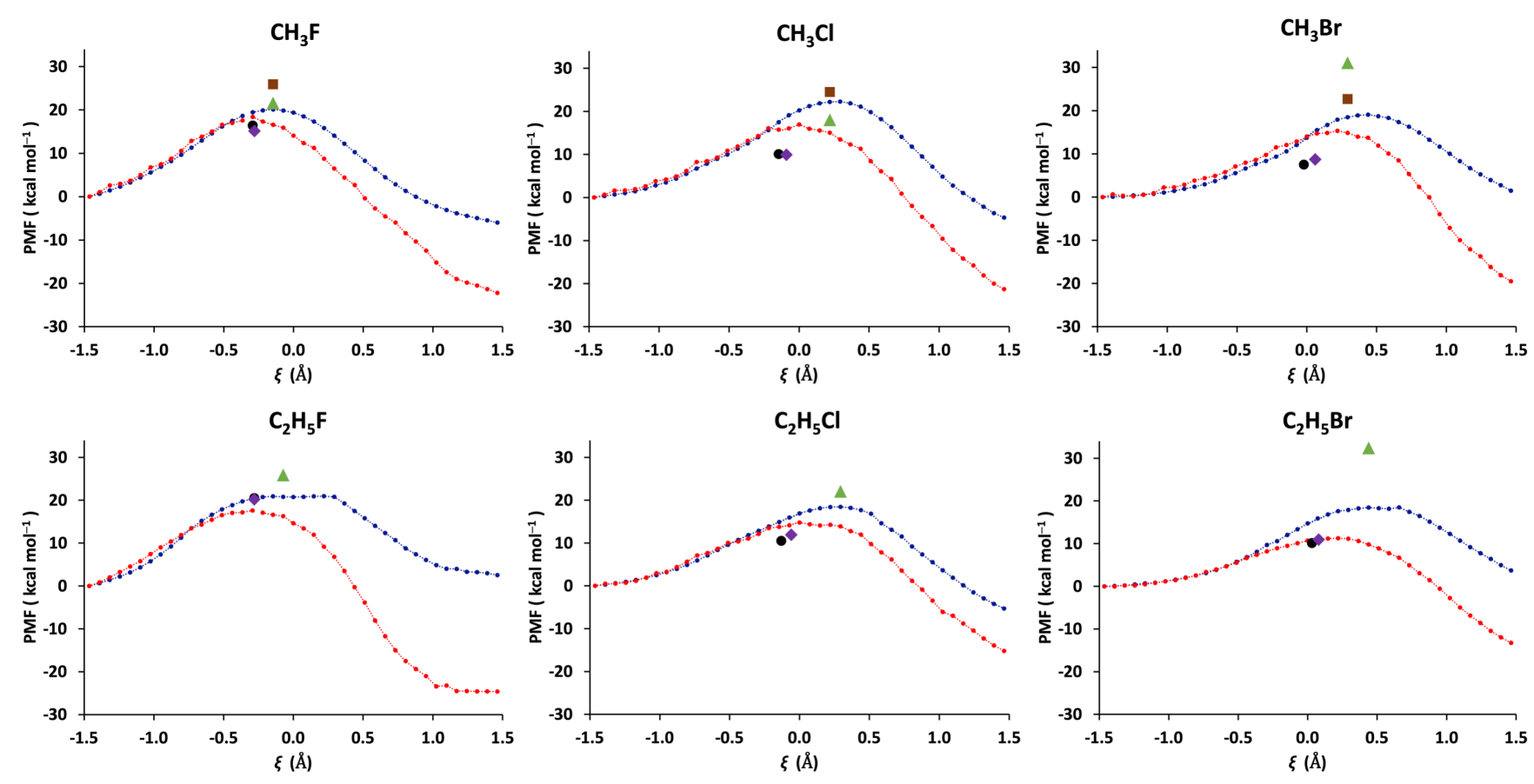

Figure 2. Potentials of Mean Force (PMFs) along the reaction coordinate $\xi$ for $\mathrm{OH}^{-}$reacting with $\mathrm{CH} 2 \mathrm{XY}(\mathrm{X}=\mathrm{F}, \mathrm{Cl}, \mathrm{Br} ; \mathrm{Y}=\mathrm{H}, \mathrm{CH} 3)$ using HES (blue) or HEB (red). Gibbs free energies using EE-NMA for HIB (black circles) or HIM (purple diamonds), EE-MCC for HES (green triangles) and experiment via TST (brown squares) of the transition state relative to the reactant encounter complex.

The TSs occur at larger values of $\xi$ for the larger halides because of the longer $\mathrm{C}-\mathrm{X}$ bond. They are slightly closer to the REC for HEB than for HES and for HIM than for HIB. The most accurate method to calculate $\Delta G$ with respect to experiment is HES PMF, whose values are only a few $\mathrm{kcal} \mathrm{mol}^{-1}$ lower. However, it predicts a slightly higher barrier for $\mathrm{CH}_{3} \mathrm{Cl}$ the $\mathrm{CH}_{3} \mathrm{~F}$. The barriers by the HEB PMF method are smaller by a few kcal mol-1 and decrease in the order $\mathrm{F}, \mathrm{Cl}$ and $\mathrm{Br}$, which is the same trend as in experiment and as reported elsewhere, ${ }^{56-59}$ and aligns with the strengths of the $\mathrm{C}-\mathrm{X}$ bonds: the bond dissociation energies of $\mathrm{C}-\mathrm{F}$ are 110 and $108 \mathrm{kcal} \mathrm{mol}^{-1}$ for the methyl and ethyl halide, respectively, of $\mathrm{C}-\mathrm{Cl}$ they are $85 \mathrm{kcal}$ $\mathrm{mol}^{-1}$ and $80 \mathrm{kcal} \mathrm{mol}^{-1}$ and of $\mathrm{C}-\mathrm{Br}$ they are 71 and $68 \mathrm{kcal} \mathrm{mol}^{-1}$. ${ }^{60}$ The lower barrier for $\mathrm{CH}_{3} \mathrm{~F}$ may be due to $\mathrm{F}^{-}$being more strongly solvated in water than other halides. ${ }^{57}$ The barriers for the ethyl halides are slightly lower for $\mathrm{Cl}$ and $\mathrm{Br}$ which is in line with the bond dissociation energies above ${ }^{60}$ but higher for $F$. The EE-NMA $\Delta G$ barriers by both the HIB and HIM methods are much lower for methyl halides but higher for ethyl fluoride and more comparable to the PMF values. This likely reflects the inaccuracy of the implicit solvent model, because water is known to raise the $\Delta G$ for these reactions relative to the gas phase..$^{56}$ It also highlights the need to explicitly include solvent, despite the greater computational cost. The HES EE-MCC barriers are in the appropriate range but are more variable and display a different trend, being lower for $\mathrm{CH}_{3} \mathrm{Cl}$ and higher for both alkyl bromides. These trends can be better understood by examining the many component quantities on which they depend, examined next.

Table 2. Enthalpy and Entropy Barriers (kcal mol${ }^{-1}$ ) of All Reactions Using the EE Methods

\begin{tabular}{lccccccc} 
& \multicolumn{4}{c}{ EE-NMA } & \multicolumn{3}{c}{ EE-MCC } \\
\cline { 2 - 8 } & \multicolumn{2}{c}{ HIB } & \multicolumn{2}{c}{$H I M$} & \multicolumn{3}{c}{ HES } \\
\cline { 2 - 8 } & $\Delta H$ & $T \Delta S$ & $\Delta H$ & $T \Delta S$ & $\Delta H$ & $T \Delta S$ & $\Delta G_{R}$ \\
\hline $\mathrm{CH}_{3} \mathrm{~F}$ & 12.5 & -4.9 & 13.4 & -3.0 & 25.6 & 4.6 & 0.6 \\
$\mathrm{CH}_{3} \mathrm{Cl}$ & 6.1 & -4.9 & 7.5 & -2.1 & 23.7 & 6.4 & 0.4 \\
$\mathrm{CH}_{3} \mathrm{Br}$ & 5.7 & -1.8 & 6.7 & -2.8 & 37.2 & 6.3 & 0.6 \\
$\mathrm{C}_{2} \mathrm{H}_{5} \mathrm{~F}$ & 18.9 & -0.8 & 17.9 & -4.0 & 26.5 & 1.2 & 0.7 \\
$\mathrm{C}_{2} \mathrm{H}_{5} \mathrm{Cl}$ & 9.4 & -2.1 & 10.0 & -1.1 & 25.9 & 4.1 & 0.7 \\
$\mathrm{C}_{2} \mathrm{H}_{5} \mathrm{Br}$ & 8.1 & -1.5 & 10.4 & -1.2 & 35.9 & 3.8 & 0.7 \\
\hline
\end{tabular}

Enthalpy and Entropy Components. The enthalpy and entropy components of the TS minus the REC are given in Table 2 for each reaction and EE method. As expected for most chemical reactions, the enthalpy 
change dominates the entropy change. $\Delta H$ clearly explains the greater $\Delta G$ in explicit solvent, especially for both the bromohalides. Table S1 shows that the QM energy is large and positive, which is consistent with the destabilization of the intramolecular dipole by water, ${ }^{56,59}$ but it is partially compensated by the QM/MM and $\mathrm{MM}$ energy which together are negative and stabilising. The vibrational energy and $\triangle P V$ contributions are small, as are the Gibbs free energies for removing the US restraint on the REC (Table 2).

$\Delta S$ displays opposing trends for the two solvent models, being negative in implicit solvent but positive in explicit solvent. An inspection of the entropy terms in Tables S2 and S3 indicates that the dominant contribution to this difference is the rotational entropy of the solvent, both vibrational and topographical. This implies that there is a weakening of solvent interactions in the TS, possibly because of the more delocalised charge, even though Table S1 indicates that the QM/MM and MM energy is stabilizing ${ }^{56,59}$ For the bromohalides, it is not clear what entropy term should compensate for their large $\Delta H$ but it is likely to be an even larger gain in solvent entropy, so necessitating a more refined approachs ${ }^{61-64}$ than that used here.

Another differing trend in entropy between the implicit and explicit solvent models evident in Table S2 is that the HIB and HIM internal entropy terms decrease in the TS but slightly increase in the HES system. This increase occurs despite the entropy reduction for all systems partly owing to the missing degree of freedom along $\xi$ and the formation of covalent bonding between the reactants. It would appear that the presence of explicit solvent may dampen this reduction, possibly along with the difference in functionals. The other weak but curious trend at the molecular level is that the translational entropy increases and rotational entropy decreases for the methyl halides, but the other way around for the ethyl halides.

\section{DISCUSSION}

The novel insights provided into reaction thermodynamics by EE-MCC come at the price of some accuracy and the need for sufficient sampling. There are sizeable errors for the HES simulations and converged values could not be obtained for the shorter and more expensive HEB simulations, even though reasonable PMFs were still produced. Evidently, it is much more difficult to obtain converged probability distributions over all molecular coordinates than just one. Figure $S 1$ illustrates how $G, T S$ and $H$ using HES vary as a function of $\xi$. The values moderately well reproduce the reaction profile but are still with errors of $\sim 5 \mathrm{kcal}$ $\mathrm{mol}^{-1}$, which, being based on $1 \mathrm{~ns}$ of sampling, are larger than the errors in Table 1 based on $10 \mathrm{~ns}$. The inhomogeneous nature of a solution means that there are many more molecules to average over per mole of solute than in a pure liquid as was done in earlier work $^{35,36}$ compounded by the slower speed of QM/MM simulations. A minimal QM region of the only reacting molecules was adopted here to minimise the slowdown but more accurate studies should include additional water molecules, particularly for the solvation of $\mathrm{OH}^{-},{ }^{65,66}$ and possibly in an adaptive scheme to account for solvent diffusion. ${ }^{67,68}$

Another problematic issue is that a PMF calculation is still needed to locate the TS and that an umbrella potential must be added to keep the system localized to the TS. However, this requirement could be alleviated by running a short series of simulations in the expected region to locate the TS followed by a longer simulation at the TS. This may be especially valuable when the PMF is difficult to converge due to a long path or there is a difficulty in identifying a suitable path. Furthermore, MCC contains a number of approximations, particularly relating to the solvent topographical entropy, in order to make tractable the calculation of the full probability distribution. However, it represents a more accurate treatment of the solvent as explicit molecules compared to a continuum model that ignores the molecular detail of the solvent and treats the solute as an ideal-gas molecule. It currently represents the state-of-the-art in liquid-phase entropy, given the limitations of more accurate methods such as inhomogeneous solvation theory ${ }^{69}$ that require many more configurations to converge higher-dimensional integrals, limiting them to small rigid molecules.

\section{CONCLUSIONS}

A new free energy method, EE-MCC, has been proposed to calculate the Gibbs free energy barriers of chemical reactions in explicit solvent using $Q M / M M$ simulations. Energy and entropy are evaluated from the system Hamiltonian and entropy using Multiscale Cell Correlation together with the system Hamiltonian. EE-MCC has been applied to six nucleophilic substitution reactions between alkyl halides and hydroxide modelled with the two QM/MM methods SCC-DFTB and B3LYP DFT. EE-MCC SCC-DFTB Gibbs free energy barriers using are in reasonable agreement with the corresponding PMF and experiment. However, accuracy is affected by the difficulty in obtaining converged entropy and energy over many molecules in an expensive QM/MM simulation. EE-MCC values are better than implicit-solvent values using NMA but this 
is primarily due to the more accurate explicit-solvent energy. EE-MCC still requires the use of a PMF to identify the TS, but its primary advantages for chemical reactions are the direct route to Gibbs free energy, as done in implicit solvent, and the insightful entropy decomposition that has not previously been available in explicit solvent chemical reactions. This capability should be valuable in liquid-phase and catalyzed reactions where entropy is expected to play a larger role more comparable to that of enthalpy in determining the kinetics of chemical reactions.

\section{ASSOCIATED CONTENT}

\section{AUTHOR INFORMATION}

\section{Corresponding Authors}

* henchman@manchester.ac.uk (RHH); sam.devisser@manchester.ac.uk (SPdV).

\section{Author Contributions}

The manuscript was written through contributions of all authors. All authors have given approval to the final version of the manuscript.

\section{Funding Sources}

Punjab Education Endowment Fund (PEEF), Pakistan for a PhD scholarship (HSA).

\section{REFERENCES}

1. Schaleger, L. L.; Long, F. A., Entropies of Activation and Mechanisms of Reactions in Solution. Adv. Phys. Org. Chem. 1963, 1, 1-33.

2. Mardirossian, N.; Head-Gordon, M., Thirty Years of Density Functional Theory in Computational Chemistry: an Overview and Extensive Assessment of 200 Density Functionals. Mol. Phys. 2017, 115, 23152372.

3. $\mathrm{Hu}, \mathrm{H} . ;$ Yang, W., Free Energies of Chemical Reactions in Solution and in Enzymes with Ab Initio Quantum Mechanics/Molecular Mechanics Methods. Annu. Rev. Phys. Chem. 2008, 59, 573-601.

4. Zhang, J.; Zhang, H.; Wu, T.; Wang, Q.; van der Spoel, D., Comparison of Implicit and Explicit Solvent Models for the Calculation of Solvation Free Energy in Organic Solvents. J. Chem. Theory Comput. 2017, 13, 1034-1043.

5. Kundi, V.; Ho, J., Predicting Octanol-Water Partition Coefficients: Are Quantum Mechanical Implicit Solvent Models Better than Empirical Fragment-Based Methods? J. Phys. Chem. B 2019, 123, 6810-6822.

6. Ren, P.; Chun, J.; Thomas, D. G.; Schnieders, M. J.; Marucho, M.; Zhang, J.; Baker, N. A., Biomolecular Alectrostatics and Solvation: a

\section{SUPPORTING INFORMATION}

Error analysis in the calculation of Gibbs free fnergy barriers; energy and entropy components of TS relative to REC, including the MCC vibrational and topographical components; energies and Gibbs free energies of the HIB and HIM optimized geometries; Cartesian coordinates of the HIB and HIM REC and TS; Figure showing $G, T S$, and $H$ calculated by HES MCC versus reaction coordinate $\xi$.

\section{ACKNOWLEDGMENT}

IT services at the University of Manchester provided the Computational Shared Facility for the simulations.

\section{ABBREVIATIONS}

QM/MM, Quantum Mechanics/Molecular Mechanics; DFT, Density Functional Theory; IEFPCM, Integral Equation Formalism Polarizable Continuum Model; SCC-DFTB, Self-Consistent Charge Density Functional based Tight-Binding; GAFF, Generalized AMBER Force Field; REC, Reactant Encounter Complex; TS, Transition State; MD, Molecular Dynamics; EE, Energy Entropy; NMA, Normal Mode Analysis; PMF, Potential of Mean Force; US, Umbrella Sampling; MCC, Multiscale Cell Correlation; TST, Transition State Theory.

Computational Perspective. Q. Rev. Biophys. 2012, 45, 427-491.

7. Tomasi, J.; Mennucci, B.; Cammi, R., Quantum Mechanical Continuum Solvation Models. Chem. Rev. 2005, 105, 2999-3094.

8. Cossi, M.; Scalmani, G.; Rega, N.; Barone, V., New Developments in the Polarizable Continuum Model for Quantum Mechanical and Classical Calculations on Molecules in Solution. The J. Chem. Phys. 2002, 117, 43-54.

9. Foresman, J. B.; Keith, T. A.; Wiberg, K. B.; Snoonian, J.; Frisch, M. J., Solvent Effects. 5. Influence of Cavity Shape, Truncation of Electrostatics, and Electron Correlation on ab Initio Reaction Field Calculations. J. Phys. Chem. 1996, 100, 16098-16104. 10. Onsager, L., Electric Moments of Molecules in Liquids. J. Am. Chem. Soc. 1936, 58, 1486-1493.

11. Marenich, A. V.; Cramer, C. J.; Truhlar, D. G., Universal Solvation Model Based on Solute Electron Density and on a Continuum Model of the Solvent Defined by the Bulk Dielectric Constant and Atomic 
Surface Tensions. J. Phys. Chem. B 2009, 113, 63786396.

12. Truhlar, D. G.; Garrett, B. C.; Klippenstein, S. J., Current Status of Transition-State Theory. J. Phys. Chem. 1996, 100, 12771-12800.

13. Laidler, K. J.; King, M. C., Development of transition-state theory. J. Phys. Chem. 1983, 87, 26572664.

14. Andrés, J.; Ayers, P. W.; Boto, R. A.; CarbóDorca, R.; Chermette, H.; Cioslowski, J.; ContrerasGarcía, J.; Cooper, D. L.; Frenking, G; Gatti C., et al. Nine Questions on Energy Decomposition Analysis. J. Comput. Chem. 2019, 40, 2248-2283.

15. van der Kamp, M. W.; Mulholland, A. J., Combined Quantum Mechanics/Molecular Mechanics (QM/MM) Methods in Computational Enzymology. Biochemistry 2013, 52, 2708-2728.

16. Quesne, M. G.; Borowski, T.; de Visser, S. P., Quantum Mechanics/Molecular Mechanics Modeling of Enzymatic Processes: Caveats and Breakthroughs. Chem.: Eur. J. 2016, 22, 2562-2581.

17. Grimme, S.; Schreiner, P. R., Computational Chemistry: The Fate of Current Methods and Future Challenges. Angew. Chem. 2018, 57, 4170-4176.

18. Bernardi, R. C.; Melo, M. C. R.; Schulten, K., Enhanced Sampling Techniques in Molecular Dynamics Simulations of Biological Systems. Biochm. Biophy. Acta. 2015, 1850, 872-877.

19. Villà, J.; Štrajbl, M.; Glennon, T. M.; Sham, Y. Y.; Chu, Z. T.; Warshel, A., How Important are Entropic Contributions to Enzyme Catalysis? Proc. Natl. Acad. Sci. 2000, 97, 11899.

20. Kazemi, M.; Åqvist, J., Chemical Reaction Mechanisms in Solution from Brute Force Computational Arrhenius plots. Na. Commun. 2015, 6, 7293.

21. Åqvist, J.; Kamerlin, S. C. L., The Conformation of a Catalytic Loop is Central to GTPase Activity on the Ribosome. Biochemistry, 2015, 54, 546556.

22. Kazemi, M.; Himo, F.; Åqvist, J., Enzyme Catalysis by Entropy Without Circe Effect. Proc. Natl. Acad. Sci. 2016, 11, 2406.

23. Åqvist, J.; Kazemi, M.; Isaksen, G. V.; Brandsdal, B. O., Entropy and Enzyme Catalysis. Acc. Chem. Res. 2017, 50, 199-207.

24. Peter, C.; Oostenbrink, C.; van Dorp, A.; van Gunsteren, W. F., Estimating Entropies from Molecular Dynamics Simulations. J. Chem. Phys. 2004, 120, 2652-2661.

25. Shojaie, F.; Dehestani, M., Vibrational Mode Analysis for the Multichannel Reaction of $\mathrm{CH} 3 \mathrm{Cl}+\mathrm{OH}$. Int. J. Quantum Chem. 2012, 112, 2450-2455.

26. Espinosa-García, J.; Coitiño, E. L.; GonzálezLafont, A.; Lluch, J. M., Reaction-Path and Dual-Level Dynamics Calculations of the $\mathrm{CH} 3 \mathrm{~F}+\mathrm{OH}$ Reaction. $J$. Phys. Chem. A. 1998, 102, 10715-10722.
27. Kim, Y.; Cramer, C. J.; Truhlar, D. G., Steric Effects and Solvent Effects on $\mathrm{S}_{\mathrm{N}} 2$ Reactions. J. Phys. Chem. A 2009, 113, 9109-9114.

28. Cai, C.; Tang, W.; Qiao, C.; Jiang, P.; Lu, C.; Zhao, S.; Liu, H., A Reaction Density Functional Theory Study of the Solvent Effect in Prototype $S_{N} 2$ Reactions in Aqueous Solution. Phys. Chem. Chem. Phys. 2019, 21, 24876-24883.

29. Kubelka, J.; Bickelhaupt, F. M., Activation Strain Analysis of $\mathrm{S}_{\mathrm{N}} 2$ Reactions at $\mathrm{C}, \mathrm{N}, \mathrm{O}$, and $\mathrm{F}$ Centers. J. Phys. Chem. A 2017, 121, 885-891.

30. Giri, S.; Echegaray, E.; Ayers, P. W.; Nuñez, A. S.; Lund, F.; Toro-Labbé, A., Insights into the Mechanism of an $\mathrm{S}_{\mathrm{N}} 2$ Reaction from the Reaction Force and the Reaction Electronic Flux. J. Phys. Chem. A 2012, 116, 10015-10026.

31. Hamlin, T. A.; Swart, M.; Bickelhaupt, F. M., Nucleophilic Substitution $\left(\mathrm{S}_{\mathrm{N}} 2\right)$ : Dependence on Nucleophile, Leaving Group, Central Atom, Substituents, and Solvent. Chem. Phys. Chem. 2018, 19, 1315-1330.

32. Ensing, B.; Klein, M. L., Perspective on the Reactions Between $\mathrm{F}-$ and $\mathrm{CH} 3 \mathrm{CH} 2 \mathrm{~F}$ : The Free Energy Landscape of the $\mathrm{E}_{2}$ and $\mathrm{S}_{\mathrm{N}} 2$ Reaction Channels. Proc. Natl. Acad. Sci. U.S.A. 2005, 102, 6755.

33. Elstner, M.; Porezag, D.; Jungnickel, G.; Elsner, J.; Haugk, M.; Frauenheim, T.; Suhai, S.; Seifert, G., Self Consistent Charge Density Functional Tight Binding Method for Simulations of Complex Materials Properties. Phys. Rev. B, 1998, 58, 72607268.

34. Miriyala, V. M.; Řezáč, J., Description of noncovalent interactions in SCC-DFTB methods. $J$. Compu. Chem. 2017, 38, 688-697.

35. Ali, S. H.; Higham, J.; Henchman, H. R., Entropy of Simulated Liquids Using Multiscale Cell Correlation. Entropy 2019, 21, 750.

36. Higham, J.; Chou, S.-Y.; Gräter, F.; Henchman, R. H., Entropy of Flexible Liquids from Hierarchical Force-torque Covariance and Coordination. Mol. Phys. 2018, 116, 1965-1976.

37. Tirado-Rives, J.; Jorgensen, W. L., Performance of B3LYP Density Functional Methods for a Large Set of Organic Molecules. J. Chem. Theory Comput. 2008, 4, 297-306.

38. Frisch, M. J.; Trucks, G. W.; Schlegel, H. B.; Scuseria, G. E.; Robb, M. A.; Cheeseman, J. R.; Scalmani, G.; Barone, V.; Mennucci, B.; Petersson, G. A., et al. Gaussian 09, Revision A.1; Gaussian, Inc.: Wallingford, CT, 2009.

39. Zhao, Y. and Truhlar, D. G., The M06 Suite of Density Functionals for Main Group Thermochemistry, Thermochemical Kinetics, Noncovalent Interactions, Excited States, and Transition Elements: Two New 
Functionals and Systematic Testing of Four M06-class Functionals and 12 Other Functionals. Theor. Chem. Acc. 2008, 120, 215-241.

40. Zhao, Y.; Truhlar, D. G., Density Functionals with Broad Applicability in Chemistry. Acc. Chem. Res. 2008, 41, 157-167.

41. D.A. Case, R. M. B., D.S. Cerutti, T.E. Cheatham, III, T.A. Darden, R.E. Duke, T.J. Giese, H. Gohlke, A.W. Goetz, N. Homeyer, et al. AMBER 2016. University of California, San Francisco. 2016.

42. Senn, H. M.; Thiel, W., QM/MM studies of enzymes. Curr. Opin. Chem. Biol. 2007, 11, 182-187.

43. Gaus, M.; Goez, A.; Elstner, M., Parametrization and Benchmark of DFTB3 for Organic Molecules. J. Chem. Theory Comput. 2013, 9, 338354.

44. Kubillus, M.; Kubař T.; Gaus, M.; Řezáč, J. and Elstner, M., Parameterization of The DFTB3 Method for $\mathrm{Br}, \mathrm{Ca}, \mathrm{Cl}, \mathrm{F}, \mathrm{I}, \mathrm{K}$, and $\mathrm{Na}$ in Organic and Biological Systems. J. Chem. Theory Comput. 2015, 11, 332-342.

45. The DFTB Website. http://www.dftb.org (accessed October 10, 2018)

46. Wang, J.; Wolf, R. M.; Caldwell, J. W.; Kollman, P. A.; Case, D. A., Development and Testing of a General Amber Force Field. J. Comput. Chem. 2004, 25, 1157-1174.

47. Wang, J.; Wang, W.; Kollman, P. A.; Case, D. A., Automatic Atom Type and Bond Type Perception in Molecular Mechanical Calculations. J. Mol. Graph. Model. 2006, 25, 247-260.

48. Lin, Y.; Pan, D.; Li, J.; Zhang, L.; Shao, X., Application of Berendsen Barostat in Dissipative Particle Dynamics for Nonequilibrium Dynamic Simulation. J. Chem. Phys. 2017, 146, 124108.

49. Götz, A. W.; Clark, M. A.; Walker, R. C., An Extensible Interface for QM/MM Molecular Dynamics Simulations with AMBER. J. Comput. Chem. 2014, 35, 95-108.

50. Torrie, G. M.; Valleau, J. P., Monte Carlo Study of a Phase-separating Liquid Mixture by Umbrella Sampling. J. Chem. Phys. 1977, 66, 14021408.

51. Kästner, J., Umbrella Sampling. Wiley Interdiscip. Rev. Comput. Mol. Sci. 2011, 1, 932-942.

52. Kumar, S.; Rosenberg, J. M.; Bouzida, D.; Swendsen, R. H.; Kollman, P. A., The Weighted Histogram Analysis Method for Free Energy Calculations on Biomolecules. I. The method. J. Comput. Chem. 1992, 13, 1011-1021.

53. Souaille, M.; Roux, B., Extension to the Weighted Histogram Analysis Method: Combining Umbrella Sampling with Free Energy Calculations. Comput. Phys. Commun. 2001, 135, 40-57.
54. Higham, J.; Henchman, R. H., Locally Adaptive Method to Define Coordination Shell. J. Chem. Phys. 2016, 145, 084108.

55. Higham, J.; Henchman, R. H., Overcoming the Limitations of Cutoffs for Defining Atomic Coordination in Multicomponent Systems. J. Comput. Chem. 2018, 39, 705-710.

56. Yin, H.; Wang, D.; Valiev, M., Hybrid Quantum Mechanical/Molecular Mechanics Study of the $\mathrm{S}_{\mathrm{N}} 2$ Reaction of $\mathrm{CH} 3 \mathrm{Cl}+\mathrm{OH}-$ in Water. J. Phys. Chem. A 2011, 115, 12047-12052.

57. Glew, D. N.; Moelwyn-Hughes, E. A.; Norrish, R. G. W., The kinetics of the Acid and Alkaline Hydrolysis of Methyl Fluoride in Water. P. Roy. Soc. Lond. A. Math. Phys. Sci. 1952, 211, 254-265.

58. Moelwyn-Hughes, E. A.; Norrish, R. G. W., The Kinetics of Certain Reactions Between Methyl Halides and Anions in Water. P. Roy. Soc. Lond. A. Math. Phys. Sci. 1949, 196, 540-553.

59. Chen, J.; Xu, Y.; Wang, D., A Multilayered Representation, Quantum Mechanical and Molecular Mechanics Study of the $\mathrm{CH} 3 \mathrm{~F}+\mathrm{OH}-$ Reaction in Water. J. Comput. Chem. 2014, 35, 445-450.

60. McMillen, D. F.; Golden, D. M., Hydrocarbon Bond Dissociation Energies. Annu. Rev. Phys. Chem. 1982, 33, 493-532.

61. Nguyen, C. N.; Kurtzman Young, T.; Gilson, M. K., Grid Inhomogeneous Solvation Theory: Hydration Structure and Thermodynamics of the Miniature Receptor Cucurbit[7]uril. J. Chem. Phys. 2012, 137, 044101.

62. Gerogiokas, G.; Southey, M. W. Y.; Mazanetz, M. P.; Heifetz, A.; Bodkin, M.; Law, R. J.; Henchman, R. H.; Michel, J., Assessment of Hydration Thermodynamics at Protein Interfaces with Grid Cell Theory. J. Phys. Chem. B 2016, 120, 10442-10452.

63. Gerogiokas, G.; Calabro, G.; Henchman, R. H.; Southey, M. W. Y.; Law, R. J.; Michel, J., Prediction of Small Molecule Hydration Thermodynamics with Grid Cell Theory. J. Chem. Theory Compu. 2014, 10, 35-48.

64. Pattni, V.; Vasilevskaya, T.; Thiel, W.; Heyden, M., Distinct Protein Hydration Water Species Defined by Spatially Resolved Spectra of Intermolecular Vibrations. J. Phys. Chem. B 2017, 121, 7431-7442.

65. Choi, T. H.; Liang, R.; Maupin, C. M.; Voth, G. A., Application of the SCC-DFTB Method to Hydroxide Water Clusters and Aqueous Hydroxide Solutions. J. Phys. Chem. B 2013, 117, 5165-5179.

66. Goyal, P.; Qian, H.-J.; Irle, S.; Lu, X.; Roston, D.; Mori, T.; Elstner, M.; Cui, Q., Molecular Simulation of Water and Hydration Effects in Different Environments: Challenges and Developments for 
DFTB Based Models. J. Phys. Chem. B 2014, 118, 11007-11027.

67. Park, K.; Götz, A. W.; Walker, R. C.; Paesani, F., Application of Adaptive QM/MM Methods to Molecular Dynamics Simulations of Aqueous Systems. J. Chem. Theory Comput. 2012, 8, 2868-2877.

68. Duster, A. W.; Wang, C.-H.; Garza, C. M.; Miller, D. E.; Lin, H., Adaptive Quantum/Molecular
Mechanics: What have we Learned, Where are We, and Where do We go from Here? WIRES Comput. Mol. Sci. 2017, 7, e1310.

69. Lazaridis, T., Inhomogeneous Fluid Approach to Solvation Thermodynamics. 1. Theory. J. Phys. Chem. B 1998, 102, 3531-3541.

\section{Potential of Mean Force}
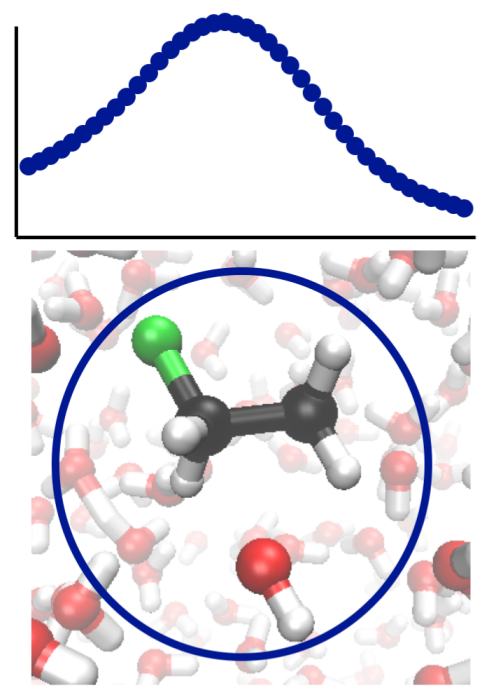

Explicit Solvent QM/MM
Energy Entropy
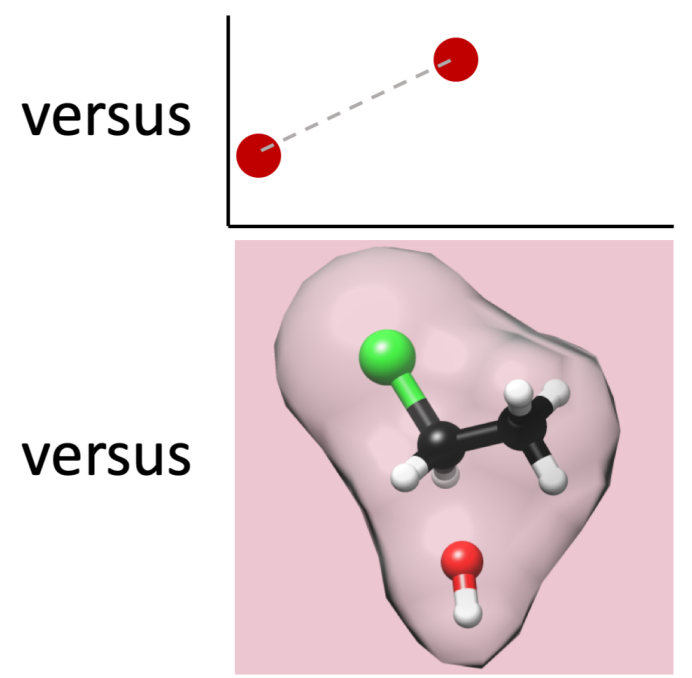

Implicit Solvent QM

Graphical Abstract 